\title{
Referate.
}

Castle, W. E. In Collaboration with H. E. Walter, R. C. Mullenix and S. Cobb. Studies of Inheritance in Rabbits. Contrib. Zoöl. Laborat. Museum. Comp. Zoöl. Harvard Coll. No. I99. Igo9. Auch: Publ. No. II4 der „Carnegie Institution of Washington"; auch No. I3 der „Papers Station Exp. Evolution“. Mit 4 Tafeln (Photographien).

Es ist nicht möglich, ganz kurz über diese wichtige experimentelle Arbeit zu berichten, da sie selbst schon sehr kondensiert und resümiert ist. Sie bildet einen gewissen AbschluB ausgedehnter Untersuchungen an Kaninchenrassen, über die der Verfasser seit I905 schon verschiedene Mitteilungen veröffentlicht hat. Die Versuche bezogen sich I. auf die Ohrenlänge, 2. auf das Körpergewicht, 3. auf die Dimensionen von Skeletteilen, 4. auf Färbung und Zeichnung.

\section{Die Ohrenlänge.}

Gerade über diesen Punkt wurde die eingehende, dokumentarische Berichterstattung des Verfassers mit Spannung erwartet, da er im Jahre I905 in vorläufigen Mitteilungen angekündigt hatte, daß die Ohrenlänge beim Kaninchen den Regeln der intermediären Vererbung (,,blending inheritance") folge und daß die intermediäre Ohrenlänge der Bastarde sich auf ihre Nachkommen vererbe. Dies blieb im Tierreich der einzige bekannte Fall intermediärer Vererbung von Varietätsbastarden mit Konstanz der Bastardform.

Die ausführliche Mitteilung der Versuchsergebnisse scheint nun die vorläufige Ankündigung einwandfrei zu bestätigen. Daß die wichtige Frage aber doch wohl noch nicht definitiv erledigt ist, soll an anderer Stelle dargelegt werden.

Castle kreuzte die bei uns unter dem Namen Widderkaninchen bekannten extrem langohrigen Formen (Ausgangsmaterial: ein einziges Paar Widder) mit gewöhlichen Kurzohren, züchtete die Bastarde mehrere Generationen weiter und machte verschiedenartige Rückkreuzungen.

Die Widderkaninchen erwiesen sich als in hohem Maße unfruchtbar. Castle macht dafür die lange fortgesetzte selektive Inzucht verantwortlich, der die Rasse ihre Entstehung verdanke. Einige Erfahrungen führen ihn zu der Vermutung, daß die Neigung zur Unfruchtbarkeit auf einem rezessiven Mendelschen Faktor beruhe.

Bei der Geburt sind die Ohren noch ganz unentwickelt, erst mit 5 bis 8 Monaten erreichen sie ihre definitive Größe. Da es nun nicht möglich war, alle Jungen des großen Zuchtmaterials großzuziehen, so machte der Verfasser statistische Erhebungen über das Wachstum der Ohren und ermittelte die Wachstumskurven für die Ohrlängen. Dies erlaubte, mit einem gewissen 
Grad von Wahrscheinlichkeit von der Ohrenlänge ganz junger Tiere auf diejenige zu schließen, die im erwachsenen Zustande erreicht worden wäre.

Die Ohrenlänge der verwendeten Kurzohren bewegte sich innerhalb der Grenzen I05-II5 cm. Reinzucht von Kurzohren ergab Konstanz der Ohrenlänge, wobei die Modifikationsbreite (etwa Io $\mathrm{mm}$ ) bei den Nachkommen nicht größer war als bei den Ausgangsformen.

Auch die Konstanz der Ohrenlänge der verwendeten extrem langohrigen Widder bei Reinzucht wurde festgestellt. Durchschnittliche Ohrenlänge etwa $220 \mathrm{~mm}$. Die Modifikationsbreite ist beträchtlicher als bei den Kurzohren, sie beträgt etwa $20 \mathrm{~mm}$ (vielleicht sogar $30 \mathrm{~mm}$ ).

Castle hebt vor Mitteilung der Kreuzungsergebnisse in besonnener Weise die möglichen Fehlerquellen und auch sehr aufrichtig die Unvollständigkeiten der Versuchsreihen hervor. Es kommt hauptsächlich folgendes in Betracht:

I. Die Ohrenlänge wird beeinflußt durch äußere Bedingungen, wie Menge und Qualität der Nahrung.

2. Die Ohrenlänge wird indirekt beeinflußt durch die Zahl der Jungen eines Wurfes, weil bei zahlreichen Jungen jedes einzelne während der Periode der Ernährung durch die Mutter weniger Nahrung bekommt, als in einem kleinen Wurfe.

3. Die Kurzohrkaninchen sind kleinere, die Widder große Kaninchenrassen. Die Wachstumsperiode der ersteren ist kürzer als die der letzteren. Messungen der Ohrenlänge, bei verschiedenen Formen in einem bestimmten Jugendalter vorgenommen, sind deshalb nicht völlig vergleichbar.

4. Blutauffrischung durch Krewzung verschiedener Rassen wird zur Entstehung von außergewöhnlich kräftigen Jungen führen, deren beträchtlichere Größe und Schwere auch eine ansehnlichere Ohrenlänge bedingen.

5. Krankheiten unterbrechen oder alterieren das normale Wachstum der Ohren.

Bei aller Vorsicht in der Verwertung des statistisch-empirischen Materials dürfen nach Castle doch folgende Ergebnisse der verschiedenen Reihen von Kreuzungsversuchen als gesichert gelten:

I. Die Kreuzung von Kaninchen mit verschieden langen Ohren ergibt eine Nachkommenschaft mit intermediärer Ohrenlänge, welche um die mittlere Ohrenlänge der Eltern pendelt oder fluktuiert. Wir wollen die sehr einfache Gesetzmäßigkeit schematisch darstellen. Die Kreuzung eines Vollblutwidders mit, nehmen wir an, $220 \mathrm{~mm}$ Ohrenlänge und eines Vollblutkurzohres, dessen Ohrenlänge $100 \mathrm{~mm}$ beträgt, ergibt $\mathrm{Halb}$ blutwidder mit $160 \mathrm{~mm}$ Ohrenlänge. Halbblutwidder untereinander gekreuzt ergibt wiederum Halbblutwidder. Halbblutwidder (supponierte Ohrenlänge $160 \mathrm{~mm}$ ) gepaart mit Vollblutwiddern (Ohrenlänge $220 \mathrm{~mm}$ ) zeugen Dreiviertelblutwidder (Ohrenlänge rgo $\mathrm{mm}$ ). Diese untereinander gekreuzt, ergeben wiederum Dreiviertelblutwidder. Einviertelblutwidder gepaart mit Vollblutkurzohren zeugen Einachtelblutwidder (Ohrenlänge II5 mm), welche bei Inzucht konstant bleiben usw. Castle hat eine sehr beträchtliche Zahl derartiger Kreuzungen vorgenommen und einen großen Teil der Resultate in 25 Tabellen übersichtlich zusammengestellt.

2. Es zeigt sich also, daß die intermediäre Ohrenlänge der Mischlinge sich bei der Weiterzucht in der Nachkommenschaft konstant erhält. Auch ist die $\mathrm{F}_{2}$-Generation nicht mehr und nicht minder variabel als die $\mathrm{F}_{1}$ Generation und von einer Mende1schen Spaltung, von einem Wiederauftreten 
der charakteristischen Ohrenlängen der großelterlichen Ausgangsformen $\left(\mathrm{P}_{1}\right.$-Generationen) zeigt sich keine Spur. Die Modifikationsbreite der Ohrenlänge der Hybriden ist meist intermediär zwischen den Modifikationsbreiten der Ohrenlängen der beiden elterlichen Typen.

3. Es hat sich auch herausgestellt, daß das Geschlecht keinen Einfluß auf die Vererbung der Ohrenlänge hat.

Für die Detailbelege müssen wir natürlich auf das Original verweisen. Wir wollen uns zur Illustration mit einigen Stichproben begnügen.

I. Beispiel. Kreuzung eines Vollblutwidderweibchens (Ohrenlänge $225 \mathrm{~mm}$ ) mit einem Vollblutkurzohrmännchen (Ohrenlänge I05 $\mathrm{mm}$ ). Mittlere Ohrenlänge der Eltern somit $165 \mathrm{~mm}$. $8 \mathrm{~F}_{1}$-Junge mit einer durchschnittlichen Ohrenlänge von $146,4 \mathrm{~mm}$. Der extremste Plusmodifikant hatte eine Ohrenlänge von $153 \mathrm{~mm}$, der extremste Minusmodifikant eine solche von $138 \mathrm{~mm}$. Modifikationsweite der Ohrenlängen der Jungen $15 \mathrm{~mm}$.

2. Beispiel. Reziproke Kreuzung. Parung eines weiblichen Vollblutkurzohrs (Ohrenlänge too mm) mit einem männlichen Vollblutwidder (Ohrenlänge $210 \mathrm{~mm}$ ). Mittlere Ohrenlänge der Eltern I55 mm. 6 Junge zeigten im Alter von 20 Wochen eine durchschnittliche Ohrenlänge von $I 48,8 \mathrm{~mm}$. Der extremste Plusmodifikant zeigte eine Ohrenlänge von $150 \mathrm{~mm}$, die extremsten Minusmodifikanten eine solche von $145 \mathrm{~mm}$.

3. Beispiel. Kreuzung eines weiblichen Vollblutwidders (Ohrenlänge $225 \mathrm{~mm}$ ) mit einem Halbblutwiddermännchen (Ohrenlänge I66 mm). Mittlere Ohrenlänge dieser Eltern I95,5 mm. 9 Junge in 2 Würfen in sehr verschiedenem Alter ( 16 Wochen bis I Jahr) auf die Ohrenlänge untersucht, zeigten eine durchschnittliche Ohrenlänge von I92,7 mm. Die Modifikationsbreite (von I80 $\mathrm{mm}$ minimaler bis $\mathrm{zu} 2 \mathrm{IO} \mathrm{mm}$ maximaler Ohrenlänge) war eine sehr beträchtliche.

4. Beispiel. Kreuzung eines weiblichen Halbblutwidders (Ohrenlänge $130 \mathrm{~mm}$ ) mit einem. männlichen Halbblutwidder (Ohrenlänge I53 mm). Mittlere Ohrenlänge dieser $2 \mathrm{~F}_{1}$-Tiere I4I,5 mm. 5 Junge der $\mathrm{F}_{2}$-Generation zeigten im Alter von 20 Wochen eine mittlere Ohrenlänge von $155 \mathrm{~mm}$. Maximale Ohrenlänge I $75 \mathrm{~mm}$, minimale I40; also eine große Modifikationsbreite. Von einer Resurrektion der beiden großelterlichen Vollbluttypen ist keine Rede!

5. Beispiel. Kreuzung von zwei Dreiviertelblutwiddern untereinander. Ohrenlängen $I 84 \mathrm{~mm}$ und $I 77 \mathrm{~mm}$. Mittel: I80,5 mm. 4 Junge zeigten im Alter von 20 Wochen Ohrlängen von I80, I85, I8o und I76 mm. Keine Andeutung einer mendelnden Spaltung!

Mit Hinblick darauf, daß eine andere als die Castlesche Deutung der intermediären Vererbung der Ohrenlänge beim Kaninchen, wie wir an anderer Stelle darlegen wollen, nicht ausgeschlossen 1st, wollen wir immerhin auf einige exzeptionelle Fäl!e aufmerksam machen. Bei der Kreuzung eines Kaninchenweibchens, dessen Ohrenlänge $130 \mathrm{~mm}$ betrug, mit einem Widdermännchen (Ohrenlänge 2 Io $\mathrm{mm}$ ), zeigte von I2 Jungen (3 Würfe) ein Exemplar im Alter von 30 Wochen eine Ohrenlänge von $200 \mathrm{~mm}$, also eine starke Annäherung an die väterliehe Ohrenlänge. Dasselbe Weibchen gebar einem Halbblutmännchen von I53 mm. Ohrenlänge unter 5 Jungen zwei, von denen im Alter von 20 Wochen das eine eine Ohrenlänge von I70, das andere eine solche von I75 $\mathrm{mm}$ zeigte, also eine Ohrenlänge, 
welche die des Elters mit den größeren Ohren ganz beträchtlich übertrifft. Castle glaubt, daß in diesem Falle die Mutter ein extremer Minusmodifikant eines Typus war, dessen mittlere Ohrenlänge viel beträchtlicher ist, daß also die Mutter nicht ihre persönliche geringere Ohrenlänge, sondern die durchschnittlich größere ihres Typus vererbte. Diese Erklärung ist zulässig, aber wie anderswo gezeigt wird, ist sie nicht die einzige.

Auch noch andere Fälle werden registriert, wo die Jungen entweder eine merklich größere Ohrenlänge aufweisen als der Elter mit dem längeren Ohr oder eine merklich geringere als der Elter mit dem kürzeren Ohr.

Die Ergebnisse seiner Kreuzungsversuche kann Castle hinsichtlich der Entstehung der Langohrrassen nur so deuten, daß es sich hier um ein Merkmal handelt, das allmählich durch Selektion gezüchtet worden ist (,which has been built up slowly as the result of selection") und nicht um eine sprungweise Mutation.

\section{Gewicht und Größe des Körpers.}

Castle bezeichnet die von ihm und seinen Mitarbeitern über diese Merkmale erzielten Resultate selbst als noch nicht befriedigend. Immerhin zeigen sie mit großer Wahrscheinlichkeit, daB auch diese quantitativen Charaktere den Regeln der intermediären Vererbung mit Konstanz der hybriden Merkmale folgen.

\section{Dimensionen der Skeletteile.}

Wahrscheinlich verhalten sich die linearen Maße der Skeletteile und ihre Proportionen bei der Vererbung intermediär. Auch ihr Gewicht und Volumen. Doch scheinen letztere Charaktere einem etwas abweichenden, noch unerkannten Gesetz zu folgen, indem sie offensichtlich in ihrer Ausprägung bei den Nachkommen stets hinter den mittleren Werten der Eltern zurückbleiben.

\section{a) Färbung und Zeichnung.}

Den ausgedehnten und planmäßigen Zuchtversuchen (Individualzucht!) über die Erblichkeitsverhältnisse von Färbung und Zeichnung, die Bateson, Castle, Crampe, Cuénot, Darbishire, Doncaster, Haacke, Hurst, Mac Curdy, Mudge, Miss Durham und neuestens Plate an Nagetieren (Ratten, Mäusen, Meerschweinchen, Kaninchen) angestellt haben, verdanken wir wohl auf zoologischem Gebiete die schönsten Bestätigungen und interessantesten Erweiterungen der Mendelschen Lehre.

In der vorliegenden Arbeit gibt Castle, ohne die detaillierten Versuchsprotokolle vorzulegen, eine Zusammenfassung der Ergebnisse seiner umfangreichen züchterischen Untersuchungen mit $\mathrm{K}$ aninchen unter gleichzeitiger Verwertung der wichtigen Hurstschen Beobachtungen. Die theoretischen Schlußfolgerungen über die genotypische Konstitution der verschiedenen Farbenvarietäten treten in der Arbeit in den Vordergrund.

Man kann nach der Farbe folgende Haupttypen, sagen wir mit Johannsen "Phänotypen" von Kaninchen unterscheiden, graue, schwarze, gelbe und weiße. Einen braunen Typus kennt Castle nicht. Innerhalb eines jeden farbigen Typus kommen abgeschwächte Pigmentierungen 
vor: blaugrau, sogenanntes Blau (abgeschwächtes Schwarz), crême (abgeschwächtes Gelb), russig Gelb usw. Sodann gibt es innerhalb eines jeden Farbentypus verschiedene erbliche Formen der Fleck- oder Plattenzeichnung. $\mathrm{Zu}$ den weißen Phänotypen (Albinos) wird auch das interessante russische oder Himalaya-Kaninchen gerechnet. Sein Auge ist rot, das Haarkleid weiß oder crême mit Ausnahme der Nase, des Oberkiefers, der Ohren, der Vorderund Hinterpfoten und des Schwanzes. Bei der Geburt sind die Russen einfarbig weiß.

Das oberste Resultat, zu dem Castle gelangte - die exakte Erblichkeitsforschung führte auch bei den andern Nagerarten zu ganz ähnlichen Ergebnissen - ist folgendes:

Die Stammfärbung sämtlicher Kaninchen ist das Wildgrau. Das Wildgrau selbst aber kommt durch das Zusammenwirken von 8 selbständigen positiven Faktoren (Farbengenen) zustande. Alle andern Färbungstypen oder Nuancen beruhen nur auf der Abschwächung oder dem völligen Fehlen eines oder mehrerer Falitoren. Ihre Bildung war und ist ein ausschließlich analytischer, retrogressiver Vorgang, eine Abbauerscheinung. Synthetisch läßt sich aus den Elementen wieder das vollständige, komplexe, ursprüngliche Wildgrau aufbauen.

Die 8 Faktoren sind folgende:

I. Ein Falstor $\mathrm{C}$ für die Bildung einer chromogenen Substanz. Es ist jener Faktor, den zuerst Cuénot in glücklicher Weise eingeführt hat, hauptsächlich zur Erklärung der Erscheinung, daß Albinomäuse verschiedene Farben ,latent" enthalten und vererben können. Der Faktor C für sich allein genügt nicht zur Pigmentbildung. Erst bei Einwirkung von Stoffen, deren Bildung durch besondere spezielle Gene bedingt wird, auf $\mathrm{C}$ entstehen die verschiedenen Farben. Fehlt der Faktor C, so findet überhaupt keine Pigmentbildung statt, und es entstehen Albinos.

Das russische oder Himalaya-Kaninchen, welches rote Augen hat und weiß odẹr crêmefarbig ist, abgesehen von den oben schon erwähnten pigmentierten Teilen, betrachtet Castle als einen partiell albinistischen Typus, dessen Ausbildung einem besondern, mendelnden Falktor $\mathrm{C}_{1}$ zuzuschreiben sei, welcher die Pigmentierung nur partiell henmt. Die Beziehungen dieses Faktors zu den übrigen, namentlich zu $\mathrm{C}$ und dem Fehlen von $\mathrm{C}$ scheinen mir aber noch nicht völlig klargelegt $\mathrm{zu}$ sein.

2. Ein Faktor $\mathrm{Y}=$,yellow", welcher zusammen mit $\mathrm{C}$ gelbe Farbe erzeugt.

3. Ein Faktor $\mathrm{Br}=$ "brown", welcher zusammen mit $\mathrm{C}$ braune Farbe hervorruft.

4. Ein Faktor $B=$,black" welcher zusammen mit $C$ schwarze Farbe erzeugt.

5. Ein Faktor $\mathrm{I}=$, ,intensity factor", welche als Verdichtungs: faktor eine starke, dichte oder konzentrierte Pigmentbildung bewirkt: es entsteht ein lebhaftes Gelb, tiefes Schwarz usw. Antagonistisch zu diesem Faktor wirkt nach Castle der rezessive Faktor $\mathrm{D}=$,,dilution factor", ein Verdünnungsfaktor, bei dessen Einwirkung das Pigment in zerstreuter oder verdünnter Form auftritt, so entsteht crême anstatt gelb, blau anstatt schwarz.

6. Ein Faktor $\mathrm{A}=$,, agouti-factor". Dieser Sprenkelungsfaktor bewirkt, daß jedes einzelne Haar gesprenkelt wird, d.h. daß das schwarze 
oder braune Pigment an jedem einzelnen Haar nur in bestimmten Zonen auftritt, Pigmentringe- oder Gürtel bildet, die mit helleren Strecken alternieren. Bei Vorhandensein dieses Faktors und der Faktoren $\mathrm{Br}$ oder $\mathrm{B}$ entsteht das wild- oder mäusegraue Haarkleid bei gleichzeitigem Fehlen des Pigmentes an der Unterseite des Rumpfes und Schwanzes.

7. Ein Faktor $\mathrm{U}=$, ,uniformity factor"“, bewirkt durch gleichmäßige Verteilung des Pigmentes über den ganzen Körper den einfarbigen $Z \mathrm{u}$ stand. Diesem Faktor steht der rezessive Faktor $\mathrm{S}=$,factor for spotting with white" gegenüber, welcher bewirkt, daß an bestimmten Strecken und Zonen sich kein Pigment bildet; ein Faktor für Scheckzeichnung oder Panachierung.

8. Ein Faktor $\mathrm{E}=$,,extension factor", welcher bloß auf Braun und Schwarz, aber nicht auf Gelb einwirkt. Diesem steht der rezessive Faktor $\mathrm{R}=$,factor for restricted distribution" gegenüber, welcher bewirkt, daß das schwarze oder braune Pigment auf die Augen und die Extremitäten beschränkt bleibt, es tritt im übrigen das gelbe Haarkleid zutage. Der Faktor $\mathrm{E}$ kommt in verschiedenen Abstufungen $\mathrm{E}^{1}, \mathrm{E}^{2}$, $\mathrm{E}^{3}$ usw. vor, denen entsprechende Modalitäten von $\mathrm{R}: \mathrm{R}^{1}, \mathrm{R}^{2}, \mathrm{R}^{3}$ gegenüberstehen. Bei der niedersten Form E finden sich am Haarkleid auf gelbem Untergrunde zahlreiche kleine braune oder schwarze Flecke.

Beziehungen $z$ wischen den Faktoren $E$ und $U$. Kommen diese Faktoren gleichzeitig vor, bei Fehlen von $\mathrm{A}$ und Vorhandensein von $\mathrm{Br}$ und B, so ist das Tier einfarbig schwarz oder wenn B fehlt (bei andern Nagetieren) braun. Ist $U$ homozygotisch durch sein Allelomorph S ersetzt, so ist das Tier schwarz oder braun mit weißen Flecken oder Zonen. Ist $\mathrm{E}$ dabei durch eine höhere Stufe, $\mathrm{E}^{1}, \mathrm{E}^{2}$ usw. ersetzt, aber $\mathrm{U}^{\mathrm{t}}$ vorhanden, so bildet das Schwarz oder Braun zunehmend größere Flecken oder Platten auf gelbern Untergrunde. Weiß fehlt dann ganz. Fehit U, wobei an seine Stelle homozygotisch $\mathrm{S}$ tritt und ist zugleich $\mathrm{E}$ durch $\mathrm{E}^{1}, \mathrm{E}^{2}$ usw. ersetzt, so wird das Haarkleid dreifarbig; auf weißem Grunde (Wirkung von S) treten gelbe und dazu noch entweder schwarze oder braune Flecken oder Platten auf. Das Tier wird dreifarbig. Niemals kommen schwarze und braune Flecken gleichzeitig mit gelb und weiß nebeneinander vor, es gibt keine vierfarbigen Typen. Es kann, wenn die Prämissen richtig sind, keine geben, weil die Faktoren E oder $\mathrm{E}^{1}$ oder $\mathrm{E}^{2}$ usw. gleichsinnig auf die Faktoren für Braun und Schwarz einwirken, so daß sich die Aus. dehnungsbezirke beider Pigmente vollkommen entsprechen, wobei die schwarze Farbe die braune vollständig verdeckt.

Castle erörtert die Frage, in welcher Weise die genotypische Konstitution der verschiedenen Färbungs- und Zeichnungstypen am prägnantesten durch Formeln ausgedrückt werden könne. Er glaubt, daß Formeln, ähnlich den in der organischen Chemie gebräuchlichen, sehr gute Dienste leisten können. Das reinrassige (komplett homozygotische), wildgraue Kaninchen, welches alle 8 oben angeführten positiven, resp. dominanten Faktoren besitzt, wiirde Gameten bilden, deren genotypische Konstitution durch folgende Formel prägnant ausgedrückt würde:

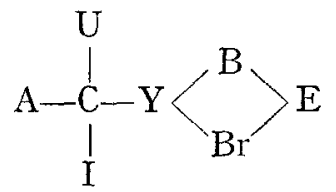


Der Typus selbst (die Zygote) bekäme nach Castle folgende Formel:

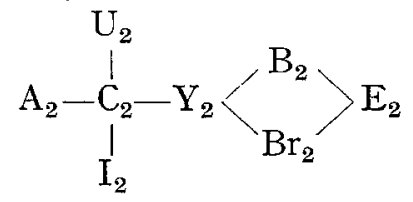

Der Index 2 gibt hier an, daß 2 identische Faktoren homozygotisch zusammengetreten sind. Die gebräuchliche Mendelsche Schreibweise ist die, daß man die Symbole doppelt schreibt: also AA (nach Castle $A_{2}$ ), CC (nach Castle $\mathrm{C}_{2}$ ) usw. Gegen die Castlesche Schreibweise ist gewiß nichts einzuwenden. Welche von beiden den Vorzug verdient, ist gewissermaßen Geschmackssache. In erster Linie wird der Gesichtspunkt der Übersichtlichkeit maßgebend sein.

Durch die obigen Formeln soll zum Ausdruck kommen, daß C, der Faktor für die chromogene Substanz, ein solcher ist, ohne den sich überhaupt keine Farbe entwickeln kann. Es sind deshalb alle andern Faktoren direkt oder indirekt mit ihm verbunden. In der Formel kommt auch zum Ausdruck, daß der Faktor E nur (in gleichsinniger Weise) auf die Faktoren $\mathrm{B}$ (schwarz) und $\mathrm{Br}$ (braun) einwirkt. Diese letzteren Faktoren ihrerseits können nur zur Geltung kommen, wenn schon Y (der Faktor für Gelb) vorhanden ist. Deshalb sind ihre Symbole ( $\mathrm{Br}$ und $\mathrm{B}$ ) durch Vermittlung von $\mathrm{Y}$ mit $\mathrm{C}$ verbunden.

Ein komplett homozygotisches einfarbig schwarzes Tier unterscheidet sich von dem komplett homozygotischen wildgrauen nur dadurch, daß der Sprenkelungsfaktor für alle einzelnen Haare, der Faktor A, ganz fehlt. Dieses Fehlen bringt Castle in der Formel dadurch zum Ausdruck, daß er das Symbol A einfach wegläßt. Die Formel für den komplett homozygotischen einfarbig schwarzen Typus wäre also

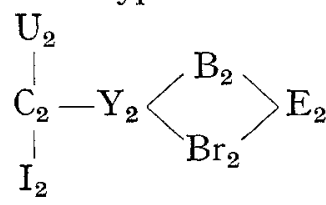

Albinos unterscheiden sich von pigmentierten Tieren dadurch, daß der Faktor C für die chromogene Substanz fehlt. Die Formel für einen komplett homozygotischen Albino mit latentem, einfarbigem Schwarz wäre also<smiles>[Y7][Y10]([Y17])[B][Ba][Ba]</smiles>

Nach der üblichen Sckreibweise würde man das Fehlen eines Faktors durch dasselbe Symbol angében, wie das Vorhandensein, aber durch kleine Buchstaben ausdrücken, so daß die vorstehende Formel für einen „sit venia" schwarzen komplett homozygotischen Albino lauten würde

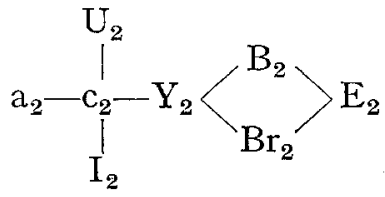


Es scheint uns, daß diese Schreibweise übersichtlicher ist, als die von Castle vorgeschlagene, da $\beta$ sie eine raschere und leichtere Orientierung bei der Ermittlung der verschiedenen Kombinationen gestattet.

Wenn zwei Gameten von ungleicher Konstitution sich vereinigen, so entsteht eine Heterozygote. Eine solche Heterozygote kann komplett oder nur partiell heterozygotisch sein. Im ersteren Falle ist jeder Faktor nur in der Einzahl vertreten, im letzteren Falle sind ein oder mehrere positive Faktoren in der Zweizahl (homozygotisch) vorhanden.

Die Formel für einen wildgrauen Typus beispielsweise, der bloß mit Bezug auf den Sprenkelungsfaktor A heterozygotisch wäre, würde nach Castle lauten

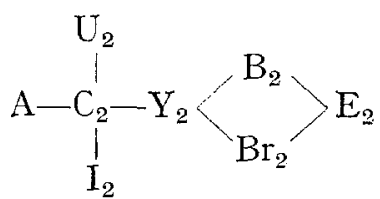

während die übliche Schreibweise wäre

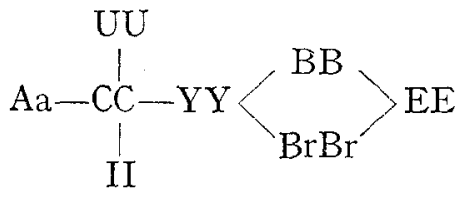

wobei A das Vorhandensein, a das Fehlen des Sprenkelungsfaktors angibt.

Die Gametenbildung erfolgt nun so, daß sämtliche Gameten, die ein Tier erzeugt, für welches diese Formel paßt, die Faktoren enthalten, welche in seiner Konstitutionsformel doppelt (homozygotisch) vorkommen, nur die Hälfte der Gameten hingegen bekommen den Faktor, der in der Konstitutionsformel in der Einzahl vorkommt, im vorliegenden Falle den Faktor A. Die andere Hälfte geht mit Bezug auf diesen Faktor leer aus. Die eine Hälfte der Gameten unseres mit Bezug auf A heterozygotischen grauen Typus hätte die Formel<smiles>[Y]C([Y])([3H])[Y]1[B][13B]C1</smiles>

und die andere Hälfte die Formel<smiles></smiles>

während nach der üblichen Schreibweise die Formel für die eine Hälfte der Gameten wäre<smiles>[Y]C([Y])([Tl])[Y]BBr</smiles> 
und für die andere<smiles>[H][Y]([CH])([Tl])[B][P]</smiles>

Nehmen wir nun an, ein Typus sei komplett heterozygotisch und führen wir das Prinzip des Vorkommens und Fehlens der Faktoren in den Formeln nach dem Castleschen System konsequent durch, so wäre cie Formel für den komplett heterozygotischen Typus folgende

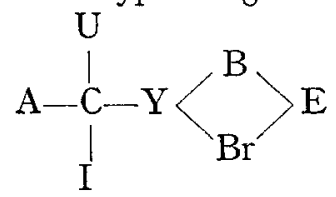

Ein Teil der Gameten (mit sämtlichen positiven Faktoren) hätte die Konstitutionsformel<smiles></smiles>

für einen andern Teil aber (mit sämtlichen negativen Faktoren) ließe sich überhaupt keine Formel aufstellen, da ihnen alle positiven Faktoren fehlen. Es läßt sich also die Castlesche Methode nicht durchführen.

Nach der üblichen Schreibweise lautet die Formel für den komplett heterozygotischen Typus

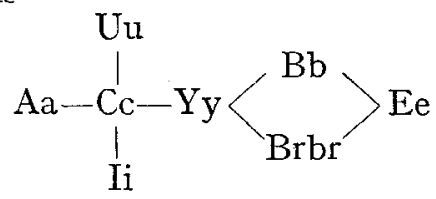

Es handelt sich um einen Fall von 8 fachem Hybridismus. Es werden 256 verschiedene Arten von reinen Gameten in gleicher Zahl gebildet, deren Formeln sich nach der üblichen Schreibweise leicht ermitteln und beurteilen lassen. Eine Art von Gameten (unter 256) enthält sämtliche positive Gene, Formel<smiles>[Y]C([Tl])([Tl])[Te]1[B][13B]1</smiles>

Eine Art von Gameten (unter 256) enthält alle negativen Gene, Formel bei der üblichen. Schreibweise

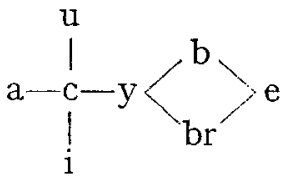


Für diese kann Castle überhaupt keine Formel aufstellen. Die Etiketten dieser Gameten könnten nur unbeschriebene Blätter sein. Allein Castle ist nicht konsequent in der Durchführung der Methode der positiven und negativen Faktoren, sondern er stellt in gewissen Paaren von Allelo. morpha einen positiven dominanten Faktor einem positiven rezessiven Faktor gegenüber. So stellt er dem positiven dominanten Faktor für Einfarbigkeit (Symbol U) den positiven rezessiven Faktor für Panachierung (Symbol S), ferner dem positiven dominanten Verdichtungsfaktor I den positiven rezessiven Verdünnungsfaktor D, sodann dem positiven dominanten „Extension factor" $\mathrm{E}$ den positiven rezessiven ,Restriction factor" $\mathrm{R}$ gegenüber. Das ginge ja schließlich noch an, wenn Castle wenigstens nach der alten bewährten Mendelschen Methode die beiden antagonistischen Merkmale, resp. ihre Faktoren, mit dem nämlichen Symbol bezeichnen würde, das dominante mit dem großen und das rezessive mit dem kleinen. Aber Castle gebraucht für die dominanten, wie für die rezessiven Faktoren große Buchstaben und für die beiden antagonistischen Merkmale eines Paares verschiedene Symbole. Es entsteht dadurch eine Konfusion und Unübersichtlichkeit, die besonders dem Ungeübten das Verständnis der so überaus wichtigen und wertvollen Abhandlung Castles erschwert und von der man nur hoffen darf, daß sie keine Nachahmung finde.

Es wäre ein Leichtes gewesen, die Constitutionsformeln nach den einheitlichen Prinzip der ,Presence and Absence Hypothesis", der positiven und negativen Faktoren, durchzuführen. Etwa in folgender Weise:

Dem Faktor für Einfarbigkeit, Symbol U, stellen wir das Fehlen dieses Faktors mit u gegenüber.

Fehlt der Faktor für Einfarbigkeit, so ist das pigmentierte Tier weiß panachiert; tritt der Faktor $U$ auf, so bewirkt er die gleichmäßige Ausdehnung der (epistatischen) Farbe (schwarz oder braun oder grau usw.) über den ganzen Körper.

Dem Verdichtungsfaktor I stellen wir sein Fehlen i gegenüber. Fehlt I, so ist das Pigment in verdünnter oder zerstreuter Form vorhanden, die Farbe dementsprechend schwach ausgeprägt. Tritt der Verdichtungsfaktor I auf, so bewirkt er eine Konzentration oder dichte Anhäufung des Pigmentes, die Farbe wird intensiv, aus dem sogenannten Blau (das in Wirklichkeit mehr ein Aschgrau ist) wird Schwarz, aus Crême Gelb, aus Zimtbraun Kastanienbraun usw.

Es ist gewiß zulässig und in manchen Fällen bequem, in der Weise, wie es Mendel tat, zwei positive Faktoren einander gegenüberzustellen, von denen der eine dominant und der andere rezessiv sein kann. Unzulässig jedoch ist es, diese Methode mit derjenigen des Vorhandenseins und Fehlens eines Faktors zu verquicken, $\mathrm{zu}$ kombinieren.

Wenn zwei Typen nur in einem Faktor verschieden sind oder wenn nur ein unterscheidender Faktor diskutiert wird, so ist das Mendelsche Verfahren sehr bequem. Kreuze ich einen schwarzen Hund mit einem braunen Hund, so kann ich die schwarze Haarfarbe als dominantes Merkmal $B$ dem braunen br gegenüberstellen. Der $F_{1}$-Generation ist schwarz und es lommt ihr die Formel zu Bbr. Die $F_{2}$-Generation besteht aus 2 Phänotypen und 4 genotypisch verschiedenen Formen; auf je 4 Exemplare kommt nämlich ein braunes brbr, ein homozygotisch schwarzes $\mathrm{BB}$ und 2 heterozygotisch schwarze Bbr und brB. - Einer ausgedehnteren Erfahrung entspricht aber diese Darstellungsweise nicht, weil erstere gezeigt hat, daß auch beim schwarzen Hund das braune Pigment vorkommt, aber als hypostatisches Pigment vom epistatischen Schwarz gänzlich verdeckt wird. 
Die Verhältnisse werden erschöpfender durch die Methode der positiven und negativen Faktoren dargestellt. Der schwarze Hund hat dann die Formel $\mathrm{BBBrBr}$, der braune die Formel bbBrBr; in der $\mathrm{F}_{1}$-Generation erhalten wir die Formel $\mathrm{BbBrBr}$ und in der $\mathrm{F}_{2}$-Generation 4 Typen, nämlich a) $\mathrm{BBBrBr}=$ homozygotisch schwarz, b) $\mathrm{BbBrBr}=$ heterozygotisch schwarz, c) $\mathrm{bBBrBr}=$ heterozygotisch schwarz und d) $\mathrm{bbBrBr}=$ homozygotisch braun, also schwarze Phänotypen und braune im Zahlenverhältnis von $3: r$, mithin dasselbe Ergebnis.

Die Methode der Gegenïberstellung von je 2 Faktoren, die beide positiv sein und zueinander im Verhältnis von Dominanz und Rezessivität stehen können, versagt aber schon dann vollständig, wenn es sich darum handelt den Fall zu erklären, daß bei Kreuzung von 2 scheinbar einheitlichen Merkmalen in der Nachkommenschaft ein drittes neues Merkmal auftritt. Kreuze ich ein wildgraues Nagetier mit einem braunen, so tritt in der $F_{1}$-Generation neben wildgrau und braun ev. gesprenkelt braun auch schwarz auf. Diese Erscheinung läßt sich mit der Methode der positiven und negativen Faktoren leicht darstellen. Der wildgraue Typus enthält drei positive, voneinander unabhängige selbständige Faktoren, die zueinander in einem bestimmten Verhältnis der Überordnung resp. Unterordnung stehen, den Faktor für braune Farbe Br, den Faktor für Schwarz B und den Faktor für die Sprenkelung A. Die Formel für den homozygotischen grauen Typus ist also AABBBrBr. Beim braunen Typus feblen die Faktoren für Schwarz und für die Sprenkelung. Die Formel dieses Typus würde also nach der "Presence and Absence"-Methode lauten aabbBrBr. Es ist nun leicht ersichtlich, daß es sich bei der Kreuzung um einen Fall von Dihy bridismus (nicht von Monohybridismus) handelt, und daß in der $\mathrm{F}_{2}$ Generation Typen auftreten müssen, in denen zwar der positive Faktor A (Sprenkelungsfaktor) fehlt, dagegen die positiven Faktoren B und Br, der erstere entweder homozygotisch oder heterozygotisch, vorkommen. Die Formeln wären also aaBBBrBr oder aaBbBrBr. Diese Typen sind aber schwarz, da das Braun von dem Schwarz epistatisch verdeckt wird.

Die Methode der Gegenüberstellung von positiven und negativen Faktoren, die von Correns und $\mathrm{C}$ nénot in ihren ersten Anfängen eingeführt und sodann hauptsächlich von Bateson, Baur und Nilsson Ehle klar begründet und konsequent durchgeführt worden ist, erscheint uns als ein wahres Kolumbusei. Sie öffnet weit den Zugang zu einer einfachen Erklärung einer Menge von komplizierten Vererbungserscheinungen bei Kreuzungen, die dadurch bedingt werden, daß bei einem Typus eine ganze Anzahl von selbständigen, spaltenden Faktoren in hierarchischer Ordnung vorkommen, von denen äußerlich nur der oberste in die Erscheinung tritt. Bei Tachea hortensis und nemoralis gibt es für jede Spezies, wenn wir nur die verschiedene Bänderung (Ausfall von Bändern, Verschmelzung von Bändern) in Betracht ziehen, 89 Bändervarietäten, von denen die meisten tatsächlich beobachtet worden sind. Es ist sicher. daß diese erbliche Variation durch zahlreiche selbständige Faktoren bedingt wird, die nebeneinander vorkommen können. So kann eine ungebänderte Form verschiedene Typen der Bänderung ,,latent" enthalten, beispielsweise einen einbändrigen, einen zweibändrigen, den fünfbändrigen Zustand usw., wobei der niedriger gebänderte Zustand immer dem höher gebänderten übergeordnet ist. Bei geeigneten Kreuzungen treten die ,latenten" Merkmale in die Erscheinung.

Die vorstehenden kritischen Bemerkungen sollen den Wert der Castleschen Untersuchung nicht im mindesten verkleinern. Es ist 
durchaus zugegeben, daß bei Annahme der Castleschen Faktoren und ihrer supponierten Wirkungsweise die zahlreich verschiedenen Erblichkeitsphänomene, die sich bei der Kreuzung der verschiedenen Typen ergeben, so gut wie restlos erklärt, $d$. h. resïmiert, zusammengefaßt, in einfachster und doch erschöpfender Weise beschrieben werden. Castle kennt nach Färbung und Zeichnung, ohne subtilere, indessen auch erbliche Nuancen der Färbung und die verschiedenen Abstufungen der ,Panachierung" zu berïcksichtigen, Ig Kaninchenvarietäten, sagen wir „Phänotypen“, von denen er mehrere durch geeignete Kreuzungen neu gezüchtet hat. Diese Phänotypen werden sich wohl alle rein, d. h. komplett homozygotisch, züchten lassen; viele sind tatsächlich rein gezüchtet worden. Daneben können sie selbstverständlich in verschiedenem Grade heterozygotisch (mono, di-, tri-, tetra-usw. -heterozygotisch) repräsentiert sein. Die komplett homozygotischen Formen bilden nur eine Art von Gameten, die monoheterozygotischen zwei Arten, die diheterozygotischen vier Arten, die triheterozygotischen acht Arten usw. Die kreuzungsanalytische Untersuchung, ob ein Vertreter eines Phänotypus homozygotisch oder heterozygotisch ist und wenn heterozygotisch dann in welchem Maße und für welche Faktoren, ist natürlich bei einer so großen Anzahl von Faktoren recht umständlich. Castle und seine Mitarbeiter haben diese Kreuzungsanalyse in großem Umfange getrieben, so daß einige Tausende von gezüchteten Kaninchen zur Verwertung kamen. Die Autoren veröffentlichen nur eine beschränkte Auswahl der Ergebnisse der mannigfaltig kombinierten Kreuzungsversuche, die vielfach großes Interesse darbieten, auf deren Besprechung wir aber verzichten müssen. Besonders wichtig sind $\mathrm{u}$. a. auch die Eigebnisse über den Albinismus bei den Kaninchen, welche eine glänzende Bestätigung der Forschungsresultate sind, zu denen Cuénot, Hurst u. a. bei verschiedenen Nagern gelangten und die sich dahin zusammenfassen lassen, daß der albinistische Phänotypus, ,im Geheimen" die ganze Musterkarte von Färbungs- und Zeichnungsvarietäten umfassen, alle möglichen Kombinationen von Färbungs- und Zeichnungsfaktoren latent enthalten kann, ohne daß genotypisch außerordentlich verschiedene Albinos äußerlich irgendwie unterschieden werden könnten. Es braucht bei irgend einer „Rasse“ nur der Faktor C in Wegfall zu kommen, so entsteht ein Albino und man braucht nur durch Kreuzung mit irgend einer pigmentierten Form diesen Faktor wieder zuzuführen, um sofort wieder in der Nachkommenschaft die durch die genotypische Konstitution bedingte Ausprägung der Färbung und Zeichnung entsprechend der theoretischen Erwartung auftreten $\mathrm{zu}$ sehen.

Und nun zum Schlusse eine Anregung, die hoffentlich auf empfänglichen Boden fallen wird. Es sind Io Jahre verflossen, seitdem die Mendelschen Regeln wieder entdeckt worden sind und seitdem hat die exakte Vererbungsforschung einen ungeahnten Aufschwung genommen. Aber es gibt im ganzen doch nur wenige Biologen, die den großen Fortschritten zu folgen vermögen. Schuld daran ist nicht zum geringsten Teile die Anarchie, die in der Terminologie und der Symbolistik auf den verschiedenen Sprachgebieten und sogar bei den verschiedenen Autoren eines und desselben Sprachgebietes herrscht. Es sollte, meine ich, nicht schwer fallen, hier Abhilfe zu treffen. Es wäre eine dankbare Aufgabe für die internationalen Kongresse, hier eine internationale Vereinbarung 
herbeizuführen. Heute wählt jeder Autor die Symbole nach den Bezeichnungen seiner Muttersprache, der Faktor für Schwarz heist englisch $B$, französisch $\mathrm{N}$, deutsch $\mathrm{S}$. Können wir uns nicht einigen, die Symbole der griechischen Bezeichnungen zu entlehnen? Können wir nicht einheitliche Bezeichnungen für die Farben einführen? Sollte es schwer sein, die Methode der positiven und negativen Faktoren ganz allgemein durchzuführen, unter Verwendung der großen und kleinen Buchstaben? Würde es nicht eine wesentliche Vereinfachung sein, wenn man für die bekannte Hierarchie von Faktoren einheitliche Zeichen einführen würde, etwa die in der Mathematik gebräulichen $>$ größer als ... und $<$ kleiner als ...? Dies nur einige wenige Beispiele.

Arnold Lang, Zürich.

Tennent, D. H. The dominance of maternal or of paternal characters in Echinoderm hybrids. - Arch. f. Entw.-Mech. 29 I9I0, S. I-I4, 2 figg. Vernon fand bei Kreuzung zweier Seeigelgattungen, daß im Winter der väterliche, im Sommer der mütterliche Einfluß überwiege und schrieb dies dem jeweiligen Reifezustand der Eier und des Spermas zu, während Doncaster die Temperatur, Herbst daneben für den Umschlag zur mütterlichen Seite einen dem Ei vor dessen Besamung gegebenen Anstoß zur Parthenogenese verantwortlich machte.

Verf. vorliegender Arbeit erkennt diese Faktoren, mit Ausnahme des den Reifezustand in Betracht ziehenden, als mitwirkend an, sieht aber den entscheidenden Faktor in einer Änderung der Alkalinität des Seewassers. Er weist insbesondere darauf hin, daß Herbst, indem er den Bastarden einen Anstoß zur Parthenogenese gab, ebenfalls mit Konzentrationserniedrigung der $\mathrm{OH}$-Ionen arbeitete, sowie, daß möglicherweise die Alkalinität auch im Meere mit der Jahreszeit wechsle. Die letztere Vermutung wird gestützt durch die von Loeb ermittelte Tatsache, daß im Meerwasser wachsende Algen es stärker alkalisch machen; dies müßte dann während der warmen Monate, als Zeit intensiverer Assimilationstätigkeit, in erhöhtem Maße der Fall sein.

Tennent verwendet die Seeigel Toxopneustes variegatus und Hipponoë esculenti, die sich ohne weiteres reziprok bastardieren lassen. Es entstehen in beiderlei Richtung (mit Hippmoë als Vater oder als Mutter) pleiotype Bastarde, die teilweise eine Mittelstellung einnehmen und teilweise Hipponoë gleichen, deren Einfluß also jedenfalls dominiert. Als Kennzeichen wurden dabei die Stäbe des Larvenskelettes angenommen, welche bei Toxopneustes einfach, bei Hipponoë gegittert sind. Das Vorhandensein von mehr als einem Stab in den Analarmen zeigt bereits den Einfluß von Hipponoë an.

Zur Zeit, als Verf. arbeitete (Juni, Juli, - Wassertemperatur 28 bis 29,5 Grad C, ähnliche Bedingungen, wie sie Vernon, Doncaster und Herbst hatten), reagierte das Seewasser deutlich alkalisch. Erhöhte er noch die Alkalinität (Zusatz von 20 Tropfen n/Io NaOH zu $40 \mathrm{~cm}^{3}$ gewöhnlichem Seewasser), so stieg das Vorwalten von Hipponoë-Charakteren weiter an, so daß sich jetzt mehr reine Hipponoë-Plutei als früher unter den aufgezogenen Larven befanden.

Erniedrigte er aber die Alkalinität (Zusatz von 20 Tropfen n/Io Essigoder Salzsäure $\mathrm{zu} 40 \mathrm{~cm}^{3}$ gewöhnlichem Seewasser), so hatte dies die Wirkung, die Larven zur Toxopneustes-Seite hin abzulenken. Nunmehr befanden sich keine oder - in anderer Versuchsreihe - nahezu keine vollkommenen Hipponoë-Plutei unter der Nachkommenschaft, wohl aber, was es 